\title{
Web-based screening and brief intervention for hazardous drinking: A double-blind randomised controlled trial
}

\author{
Kypros Kypri, PhD ${ }^{1 *}$ \\ John B. Saunders, $\mathrm{MD}^{3}$ \\ Sheila M. Williams, DSc ${ }^{2}$ \\ Rob O. McGee, $\mathrm{PhD}^{2}$ \\ John D. Langley, PhD ${ }^{1}$ \\ Martine L. Cashell-Smith, BCom ${ }^{1}$ \\ Stephen J. Gallagher, PhD ${ }^{1}$ \\ ${ }^{1}$ Injury Prevention Research Unit \\ ${ }^{2}$ Department of Preventive and Social Medicine \\ University of Otago Medical School \\ PO Box 913, Dunedin, New Zealand \\ ${ }^{3}$ Centre for Drug and Alcohol Studies \\ Department of Psychiatry \\ School of Medicine \\ University of Queensland \\ Brisbane Qld 4029 Australia
}

* Corresponding author

School of Medical Practice and Population Health

University of Newcastle

Locked Bag 10 Wallsend NSW 2289 Australia

Phone: +61 249246205

Fax: $\quad+61249246215$

E-mail: kypros.kypri@newcastle.edu.au 


\begin{abstract}
Background: Strong evidence exists for the efficacy of screening and brief intervention for reducing hazardous drinking. However, problems have been highlighted with respect to its implementation in healthcare systems, not least of which is a reluctance of some doctors to proactively discuss alcohol with their patients.
\end{abstract}

Aims: to determine the efficacy of a novel web-based screening and brief intervention (e-SBI) to reduce hazardous drinking.

Design: a double-blind randomised controlled trial.

Setting: a university student health service.

Participants: 167 students (17-26 years) were recruited in the reception area and completed a 3-minute web-based screen including the AUDIT questionnaire. Of these, 112 tested positive, and 104 (52 females) who consented to follow-up were included in the trial.

Measurements: drinking frequency, typical occasion quantity, total volume, heavy episode frequency (females $>80 \mathrm{~g}$ ethanol, males $>120 \mathrm{~g}$ ethanol), number of personal problems, an academic problems score.

Intervention: Participants were randomised to 10-15 minutes of web-based assessment and personalised feedback on their drinking (intervention, $n=51$ ), or to a leaflet-only control group $(n=53)$.

Findings: Mean baseline AUDIT scores for control and intervention groups were $16.6(\mathrm{SD}=6.0)$ and $16.6(\mathrm{SD}=5.7)$. At six weeks, participants receiving e-SBI reported significantly lower total consumption (geometric mean ratio $=0.74 ; 95 \%$ 
In press 2004 - Addiction

confidence interval: 0.56 to 0.96$)$, lower heavy episode frequency $(0.63 ; 0.42$ to 0.92), and fewer personal problems (0.70; 0.54 to 0.91$)$. At six months personal problems remained lower $(0.76 ; 0.60$ to 0.97$)$, although consumption did not differ significantly. At six months, academic problems were lower in the intervention group relative to controls $(0.72 ; 0.51$ to 1.02$)$.

Conclusions: e-SBI reduced hazardous drinking among university students, to an extent similar to that found for practitioner-delivered brief interventions in the general population. e-SBI offers promise as a strategy to reduce alcoholrelated harm in a way that is non-intrusive, appealing to the target group, and capable of being incorporated into primary care. Research is required to replicate the findings, to determine the duration of intervention effects, and to investigate the mechanisms by which the intervention operates. 
In press 2004 - Addiction

Hazardous drinking is a leading cause of mortality and morbidity in developed regions and is an increasing problem in developing regions (Murray and Lopez, 1996; World Health Organization, 2002). In an era in which state controls on the availability of alcohol are decreasing, interventions to reduce demand for alcohol that are effective and deliverable to many are needed.

One such approach is screening and brief intervention (SBI), involving the systematic identification of people with hazardous drinking, and the provision of brief advice on how to reduce this behaviour. The most recent of six metaanalyses of opportunistic SBI, which examined the outcomes of 34 randomised controlled trials, revealed significant reductions in consumption and alcoholrelated problems (Moyer et al., 2002). These effects were seen for at least 12 months (Moyer et al., 2002) and in one US study the benefits, in terms of reductions in hospitalisation and emergency department visits, were evident four years after intervention (Fleming et al., 2002).

Despite these results, SBI is not yet routine in primary healthcare in any country. Various problems have been highlighted with respect to its implementation in healthcare systems (Aalto et al., 2003), not least of which is a reluctance of some doctors to proactively discuss alcohol with their patients (Beich et al., 2002). Research conducted with university students, a group with high levels of hazardous drinking (Kypri et al., 2002), suggested that they would be unlikely to engage in a discussion about their drinking with a healthcare professional, which 
In press 2004 - Addiction

was not self-initiated (Kypri, 2002). That research also suggested that web-based assessment and personalised motivational feedback (known generically as electronic screening and brief intervention, e-SBI) would be more acceptable to students (Kypri et al., 2003).

e-SBI has certain advantages over practitioner-delivered SBI: it involves little or no clinician contact, it can be conducted anonymously, and it can be accessed without limitations of distance. Additionally, e-SBI may be less threatening to hazardous drinkers than a face-to-face intervention. While various computerized methods for delivering SBI have been developed (Cunningham et al., 2000; Skinner et al., 2001), there have, to date, been no rigorous efficacy trials of these interventions published in the scientific literature. The aim of this study was to determine the efficacy of e-SBI in reducing hazardous drinking among university students.

\section{Methods}

Study design

The study was a double-blind randomised controlled trial. Students screening positive for hazardous drinking received either web-based assessment and personalised feedback on their drinking (intervention), or were assigned to a control group which received a leaflet on the health effects of alcohol. Ethical approval was granted by the University of Otago Ethics Committee. 
In press 2004 - Addiction

Recruitment, screening, randomisation, intervention

Users of the Student Health Service of the University of Otago, in the period 1322 March 2002 were recruited. After checking-in at reception, students aged 1726 years were invited to participate using a two-stage consent procedure. In the first stage, students were provided with an information sheet describing the study, ostensibly a series of surveys on student alcohol use and health. Those who consented were led by a research assistant to a computer, and were asked to follow on-screen instructions. In accordance with ethical approval, participants were not informed that the study was a randomised controlled trial. The target sample size was based on similar studies of practitioner-delivered SBI reviewed by Moyer et al. (2002). Accordingly, we sought to recruit at least 100 individuals, with a minimum of 50 in each group.

Participants scoring 8 or more on the AUDIT (Alcohol Use Disorders Identification Test)(Saunders et al., 1993) and consuming more than $4 / 6$ standard drinks (females/males) on one or more occasions in the preceding 4 weeks, were randomly assigned by computer to either the control group or the intervention. Randomisation was effected by computer in blocks of 10 (5 control, 5 intervention), such that approximately equal numbers of participants could be recruited in a short period. Recruitment was conducted separately by sex, to ensure approximately equal numbers of men and women.

\section{Blinding}


In press 2004 - Addiction

The research staff involved in the trial were not informed of participants' group allocation during intervention or at follow-up. The generation of the sequence and loading of it into the server database were conducted by staff who were not involved in the implementation of the trial on site. That is, they never came into contact with study participants.

Participants assigned to the control condition were presented with a web page thanking them for their involvement in the study. The research assistant gave the participant an Alcohol Facts and Effects leaflet and then initiated the second stage of the consent process, by asking for contact details to be provided for the purpose of 6-week follow-up. Upon completion, the participant was thanked and he or she resumed the wait to be seen by medical staff.

Participants assigned to the intervention condition were presented with further assessment items and then personalised feedback. Upon completion, a 'thank you' message appeared on screen. The research assistant then presented the Alcohol Facts and Effects leaflet and initiated the second stage of the consent process, as per the controls.

It should be noted that the second stage consent procedure was conducted after participants had completed the web pages used in either the intervention or control condition. Students were asked whether they were willing to complete a web-based follow-up assessment six weeks later, and to provide contact details 
In press 2004 - Addiction

for this purpose. If they did not provide contact details they were considered to have declined to give consent, and they were not included in the trial. We used their baseline data (consisting of their gender, age, ethnicity, and responses to the AUDIT) to determine if there were systematic differences between consenters and non-consenters.

It should be noted that the web pages were presented as a seamless series for both groups, and that the study had been introduced to participants as a series of surveys of their alcohol use, not an intervention trial. Thus it was intended that participants would not realise they had been assigned to one of two possible groups, or that their experience of the survey was different to that of other participants in the study. Upon completion of the final follow-up assessment, informant interviews were conducted with 10 participants (five intervention, five control) to examine whether participants were cognisant of the actual study design.

It may be argued that researchers could not have been fully blind to the study group allocation, given the different completions times for intervention and control group participants (completion times are summarised in the results section). It should, however, be noted that there was considerable variation in completion times within each group; that research assistants were operating in a busy reception environment; were responsible for managing the involvement of two or more participants simultaneously; and did not manually record 
In press 2004 - Addiction

completion times (this was effected by the server). Thus it was unlikely that research assistants could have accurately noted the likely group membership of participants. Furthermore, at the start of the study, research assistants were discouraged from trying to discover the group allocation.

\section{Intervention}

Those assigned to intervention were presented with assessment questions and then personalised feedback which together comprised the intervention.

Assessment included a 14-day retrospective drinking diary, self-reported weight, and perceptions of peer drinking norms (Kypri and Langley, 2003). Feedback consisted of a summary of recent consumption; their risk status, comparison of their consumption with recommended upper limits, and an estimate of their blood alcohol concentration for their heaviest drinking occasion in the preceding 4 weeks (criterion feedback); comparison of their consumption with that of national and university norms (normative feedback); and correction of norm misperceptions. The instrument can be viewed at http://ipru.otago.ac.nz/sbi1demo.

\section{Follow-up assessment}

Participants were invited by letter to complete a 6-week follow-up survey by clicking on a hyperlink to the site, sent to their e-mail address. Embedded in the hyperlink was a unique identifier which gained the participant access to their record via a web interface. Included with the letter was a lunch voucher valued at NZ\$4.95, as a token of appreciation for their participation. 
In press 2004 - Addiction

A reminder e-mail was sent to participants who did not respond, followed by a reminder telephone call. The 6-week follow-up phase was completed by 6 June 2002. A short pen-and-paper questionnaire posted with an invitation letter was used for follow-up at 6 months (8 September 2002). E-mail, telephone and posted reminders were used until the completion of follow-up (15 October 2002).

\section{Outcome measures}

Six outcome measures were selected:

1. Frequency of drinking: number of drinking days in the preceding 2 weeks;

2. Typical occasion quantity: number of standard drinks consumed per typical drinking occasion in the preceding 4 weeks;

3. Total volume: number of standard drinks consumed in the preceding 2 weeks;

4. Frequency of very heavy episodes: number of occasions in the preceding 2 weeks where a threshold of $80 / 120 \mathrm{~g}$ ethanol was breached, for women/men respectively;

5. Personal, social, sexual, and legal consequences of episodic heavy drinking: number of items endorsed on the Alcohol Problems Scale (APS: range 0-14) (Kypri, 2002);

6. Consequences related to academic performance: a score on the Academic Role Expectations and Alcohol Scale (AREAS: range 0-35) (Kypri, 2002). 
In press 2004 - Addiction

Outcomes 1,3 , and 4 were measured with a retrospective diary, in which the number of standard drinks was recorded for each of the preceding 14 days. Outcome 2 was measured with the question: “How many drinks containing alcohol did you have on a typical day when you were drinking in the last 4 weeks?".

The APS was created for this study, and consists of 14 items with yes/no answers, encompassing a range of problems arising from episodic heavy drinking among tertiary students (Kypri, 2002). The reference period for the problems was the preceding 4 weeks.

The AREAS consists of nine items (Kypri, 2002). For items 1-8, respondents were asked to indicate the number of times each of the events occurred in the preceding 4 weeks. These were coded as 0 "Not at all" through to 4 "Four or more times". In item 9, a self-rating of the effect of drinking on learning or grades, was scored 0 "Not at all" through to 3 "A great deal".

Coefficient alpha was calculated to measure the internal consistency of each scale (Nunnally, 1978). For the APS, alpha was 0.67 for the entire scale, and ranged from 0.62 if item 4 was excluded ("Heated argument") to 0.68 if item 12 was excluded ("Vandalism"). For the AREAS, alpha was 0.78 for the entire scale, and ranged from 0.71 if item 1 was excluded ("Late for class") to 0.79 if item 6 was excluded ("Missed practice or training"). All of the tested items were retained in 
In press 2004 - Addiction

each of the two scales. These analyses confirmed that the APS and AREAS have acceptable levels of internal consistency, making them suitable measures for the evaluation of intervention effects.

\section{Analysis}

Outcomes 1-5 were analysed using negative binomial regression for panel data. This took into account the over-dispersion in the data, which were based on counts. For outcome 6, which is a scale, we used linear regression analysis for panel data, after a log transformation. The models adjusted for baseline differences by including the AUDIT score. They also included terms for the group, follow-up assessment, and their interaction, using the $x$ tnbreg procedure in STATA (Altman and Bland, 2003). The interaction term tested for differences in the intervention effect between the two follow-up assessments. The results are presented as the ratio of the geometric mean for the intervention group to that of the control group for each follow-up assessment (Altman and Bland, 2003). Effect sizes were computed for each outcome according to the method described by Rosenthal (1991). Retrospective power analyses based on a two-tailed assumption and $a=0.05$, were conducted according to the method described by Dupont and Plummer ( 1990).

In the 6-month assessment questionnaire, participants were asked to indicate whether they were willing to participate in an informant interview concerning the research processes in return for a music voucher valued at $\$ 15.00$. The 20 
In press 2004 - Addiction

volunteers (10 control, 10 intervention) with the highest AUDIT scores were sent written invitations to participate. The first five to reply from each experimental condition (total $\mathrm{n}=10$ ) participated in a structured informant interview lasting 3040 minutes. One domain of questioning concerned participants' perceptions of what we were seeking to study, their knowledge of the study design, and specifically whether they were aware that they had been randomly assigned to one of two experimental conditions.

\section{Results}

Of 195 students checked for eligibility, 178 were invited to participate and 167 completed screening (94\%). Of these, 112 (67\%) screened positive for hazardous drinking. Eight declined further involvement (second stage consent), leaving 104 individuals in the trial (53 control, 51 intervention). The median completion time for the intervention group was 11.2 minutes. For controls, the median completion time (i.e. for screening alone) was 3.4 minutes.

The mean AUDIT score (and 95\% confidence interval) of the eight individuals who did not give second stage consent was 15.0 (11.0 to 19.0), while that of the 104 individuals who gave second stage consent was 16.6 (15.5 to 17.7). Table 1 presents summary data for the control and intervention groups. 
In press 2004 - Addiction

Participant flow and follow-up

Flow of participants through the trial is illustrated in Figure 1. At 6 weeks, follow-up assessment data were obtained from 83 participants (41 control, 42 intervention). At 6 months, data were obtained from 94 participants (47 control, 47 intervention). There were non-significant differences in mean AUDIT scores between those who did and did not complete the 6-week (mean difference $=1.2$ points; $95 \%$ confidence interval: -1.6 to 4.0$)$ and 6-month (1.8 points; $-2.0,5.6)$ follow-up assessments.

$<$ Figure 1>

Table 2 presents the median and range for the six outcomes at 6-week and 6month follow-up. Estimates of the effect of the intervention for each outcome are presented in Table 3, as the ratio of the geometric mean of the intervention group to that of the control group. At 6 weeks, relative to controls, the intervention group reported significantly lower total consumption, fewer heavy episodes, and alcohol-related problems. For quantity consumed per typical occasion, both groups reported reductions at 6 months relative to 6 weeks (ratio of the geometric means $=0.75 ; 95 \%$ confidence interval: 0.71 to 0.91$)$. At 6 months personal problems remained lower, even though reductions in consumption were no longer significant. 
For the purpose of comparison with meta-analytic review studies of brief intervention trials, effect sizes were computed for an aggregate of alcohol consumption (outcomes 1-4) and for alcohol-related problems (outcomes 5-6). These are presented in the lower part of Table 3.

$<$ Table $3>$

\section{Power analysis}

For the 6-week effect sizes reported in Table 3, with the sample sizes of this study, power (1- $\beta$ ) was 0.43 for consumption measures and 0.52 for alcoholrelated problems. For 6-month effects, the corresponding power estimates were 0.11 and 0.56 .

\section{Informant interviews}

All 10 informants indicated that they thought the study concerned either individual differences in patterns of alcohol consumption, changes in consumption over time, or both. Their reports indicated that they considered the design to be longitudinal. When asked if they knew that they had been randomly assigned to an experimental condition, none of the participants reported any awareness of this.

\section{Discussion}

At 6 weeks, the intervention resulted in reductions of $26 \%$ in total consumption, $37 \%$ in very heavy episodes, and 30\% in alcohol-related personal, sexual, and 
In press 2004 - Addiction

legal problems. At 6 months, there were reductions of $24 \%$ in alcohol-related personal, sexual, and legal problems and $28 \%$ in consequences related to academic role expectations. Although not all of the comparisons were statistically significant, all but one of the effects (outcome 2) followed the same pattern. For typical occasion consumption, the groups were similar at 6 months, the controls apparently reducing their consumption subsequent to 6-week assessment. The trial was conducted with participants and research staff blind to group allocation. The blinding was maintained across the follow-up data collection phases. Attrition was low relative to other studies of SBI (Edwards and Rollnick, 1997). Statistical power was modest for the six-week effects and low for six-month effects.

For self-reported alcohol consumption, the likelihood of under-reporting is reduced when assessment procedures minimise the potential cost of an honest response (Babor et al., 2000; Dillman, 2000). In the present study participants were assured of confidentiality, no names were used and judgmental language was avoided. Given the low sensitivity of blood markers to episodic heavy drinking (Babor et al., 2000), the typical drinking pattern among young people, reliance on self-report was necessary. It is possible that intervention group members underreported their drinking to a greater extent than did control group members, having been provided with personalised feedback at an earlier stage. If this occurred, the efficacy of the intervention would be overestimated. 
In press 2004 - Addiction

The results of this trial are most usefully placed in the context of SBI research in the general population. As was shown by Moyer et al. (2002), $\leq 3$ month effect sizes (and 95\% confidence intervals) for interventions targeting individuals not seeking treatment, were 0.67 (0.39 to 0.95$)$ for alcohol consumption measures and 0.30 (0.08 to 0.52$)$ for measures of alcohol-related problems. The e-SBI effect sizes demonstrated here fall within the confidence intervals for the Moyer et al. estimates.

For 3-6 month outcomes, Moyer et al. (2002) estimated effect sizes of 0.16 (0.10 to 0.22) for alcohol consumption measures, and 0.14 (0.08 to 0.21$)$ for alcohol-related problems. e-SBI effect sizes suggest similarly modest impacts on consumption but markedly larger decrements in alcohol-related problems (effect size $=0.44)$. It can therefore be concluded that, in the short to medium term, for university students, e-SBI performed as well as practitioner-delivered brief interventions have in non-student populations.

A significant strength of the study was the naturalistic setting of the trial. Previous studies of brief intervention for university or college students have occurred in artificial conditions, e.g. undergraduate psychology classes (Walters et al., 2000), or with highly self-selected participants (Baer et al., 1992), such that results may generalize poorly to the settings in which brief intervention could be delivered in a sustainable manner. 
In press 2004 - Addiction

Like several other studies of brief, opportunistic interventions (Moyer et al. 2002), the aim of this study was to determine whether e-SBI reduced alcohol consumption and related problems relative to no intervention. This is a population group with a high prevalence of hazardous drinking (Kypri and Langley, 2003; Kypri et al., 2002), which experiences (McGee and Kypri, under review) and inflicts high levels of alcohol-related harm (Langley et al., 2003). If it is possible to engage university students with e-SBI, there may be considerable population-level benefit of even small reductions in risk if the intervention reaches a large number of individuals.

Accordingly, a challenge of the research is to be able to measure modest effects. In addition to recruiting larger study samples it is important to maximise the contrast between intervention and control conditions to avoid Type II error. We attempted to do this by minimising the amount of baseline measurement the control group was exposed to: the AUDIT plus a few items, given that assessment itself is known to have moderating effects on alcohol consumption.

However, it is possible that assessment effects occurred at follow-up. In the process evaluation which followed the present trial, a number of control group participants suggested that the six-week assessment had a moderating effect on them. It is possible that the reduction in effect sizes at six months for consumption measures seen in this study was partly a result of the partial exposure of the control group to the intervention at six weeks. This hypothetical 
In press 2004 - Addiction

'assessment reactivity' (a Hawthorne Effect) may feature in other SBI studies and is worthy of experimental study.

The theoretical mechanisms of self-focusing feedback, the creation of discrepancy between goals and actual behaviour, and normative comparison, are some of the potential causal agents behind the effects seen here (Miller and Rollnick, 1991). However, it is also possible that non-specific effects are at work (Miller and Rollnick, 1991), for example, the greater attention given to the intervention group, measured as more time at the computer relative to controls. To examine these questions concerning mechanism, larger, more complex studies are required. Multi-arm experimental designs could be utilised to determine the relative efficacy of different types and formats of feedback (e.g., non-directive feedback versus direct advice; normative versus criterion feedback). Some form of placebo activity could be utilised to control for differences in the duration of the exposure to the computer program. It has also been suggested that there may be benefit in conducting a considerably larger trial allowing sufficient power for multivariate analysis of correction of norm misperception as a potential mediating variable in the reduction of hazardous drinking (Kypri and Langley, 2003).

For now, the results of this small trial, taken in the context of the body of work on SBI, suggest that e-SBI shows promise as an intervention for reducing hazardous drinking among university students. The authors are presently 
In press 2004 - Addiction

seeking to replicate the findings in a larger trial, with control for potential assessment reactivity, analysis of underlying mechanisms, and longer follow-up.

\section{Acknowledgements}

The authors are grateful to Dr. Jim Jerram and the Student Health Service of the University of Otago for facilitating the research. This research was funded by the Alcohol Advisory Council of New Zealand and the Health Research Council of New Zealand. 


\section{References}

Aalto, M., Pekuri, P. and Seppa, K. (2003) Obstacles to carrying out brief intervention for heavy drinkers in primary healthcare: a focus group study. Drug and Alcohol Review 22, 169-173.

Altman, D. G. and Bland, J. M. (2003) Interaction revisited: the difference between two estimates. Bmj 326, 219.

Babor, T. F., Steinberg, K., Anton, R. and Del Boca, F. (2000) Talk is cheap: Measuring drinking outcomes in clinical trials. Journal of Studies on Alcohol 61, 55-63.

Baer, J. S., Marlatt, G. A., Kivlahan, D. R., Fromme, K., Larimer, M. E. and Williams, E. (1992) An experimental test of three methods of alcohol risk reduction with young adults. Journal of Consulting and Clinical Psychology 60, 974-979.

Beich, A., Gannik, D. and Malterud, K. (2002) Screening and brief intervention for excessive alcohol use: qualitative interview study of the experiences of general practitioners. BMJ 325, 870 .

Cunningham, J. A., Humphreys, K. and Koski-Jannes, A. (2000) Providing personalized assessment feedback for problem drinking on the Internet: A pilot project. Journal of Studies on Alcohol 61, 794-8.

Dillman, D. A. (2000) Mail and internet surveys : the tailored design method. J. Wiley, New York.

Dupont, W. and Plummer, W. (1990) Power and Sample Size Calculations: A Review and Computer Program. Controlled Clinical Trials 11, 116-128.

Edwards, A. G. and Rollnick, S. (1997) Outcome studies of brief alcohol intervention in general practice: the problem of lost subjects. Addiction 92, 1699-704.

Fleming, M. F., Mundt, M. P., French, M. T., Manwell, L. B., Stauffacher, E. A. and Barry, K. L. (2002) Brief physician advice for problem drinkers: longterm efficacy and benefit-cost analysis. Alcohol Clin Exp Res 26, 36-43.

Kypri, K. (2002) Tertiary student hazardous drinking: Epidemiology and development of a brief intervention trial. PhD thesis, University of Otago, Dunedin.

Kypri, K. and Langley, J. D. (2003) Perceived norms and their relation to university student drinking. Journal of Studies on Alcohol 64, 829-834.

Kypri, K., Langley, J. D., McGee, R., Saunders, J. B. and Williams, S. (2002) High prevalence, persistent hazardous drinking in New Zealand tertiary students. Alcohol and Alcoholism 37, 457-64.

Kypri, K., Saunders, J. B. and Gallagher, S. J. (2003) Acceptability of various brief intervention approaches for hazardous drinking among university students. Alcohol and Alcoholism 38.

Langley, J. D., Kypri, K. and Stephenson, S. (2003) Secondhand effects of alcohol use among university students: Computerised survey. British Medical Journal 327, 1023-4. 
McGee, R. and Kypri, K. (in press) Alcohol-related problems experienced by university students in New Zealand. Australian and New Zealand Journal of Public Health.

Miller, W. R. and Rollnick, S. (1991) Motivational interviewing : preparing people to change addictive behavior. Guilford Press, New York.

Moyer, A., Finney, J. W., Swearingen, C. E. and Vergun, P. (2002) Brief interventions for alcohol problems: A meta-analytic review of controlled investigations in treatment-seeking and non-treatment-seeking populations. Addiction 97, 279-92.

Murray, C. J. L. and Lopez, A. D. (1996) The Global burden of disease : a comprehensive assessment of mortality and disability from diseases, injuries, and risk factors in 1990 and projected to 2020. Harvard School of Public Health on behalf of the World Health Organization and the World Bank, Cambridge, MA.

Nunnally, J. C. (1978) Psychometric theory. McGraw-Hill, New York.

Rosenthal, R. (1991) Meta-analysis: A Review. Psychosomatic Medicine 53, 247-271.

Saunders, J. B., Aasland, O. G., Babor, T. F., de la Fuente, J. R. and Grant, M. (1993) Development of the Alcohol Use Disorders Identification Test (AUDIT): World Health Organisation Collaborative Project on Early Detection of Persons with Harmful Alcohol Consumption--II. Addiction 88, 791-804.

Skinner, H., Maley, O., Smith, L., Chirrey, S. and Morrison, M. (2001) New frontiers: Using the Internet to engage teens in substance abuse prevention and treatment. In Adolescents alcohol and substance abuse: Reaching teens throuch brief interventions, Monti, P. M., Colby, S. M. and O'Leary, T. A. eds. Guildford Press, New York.

Walters, S. T., Bennett, M. E. and Miller, J. H. (2000) Reducing alcohol use in college students: A Controlled trial of two brief interventions. Journal of Drug Education 30, 361-372.

World Health Organization (2002) The World Health Report 2002, Reducing Risks, Promoting Healthy Life. WHO, Geneva. 


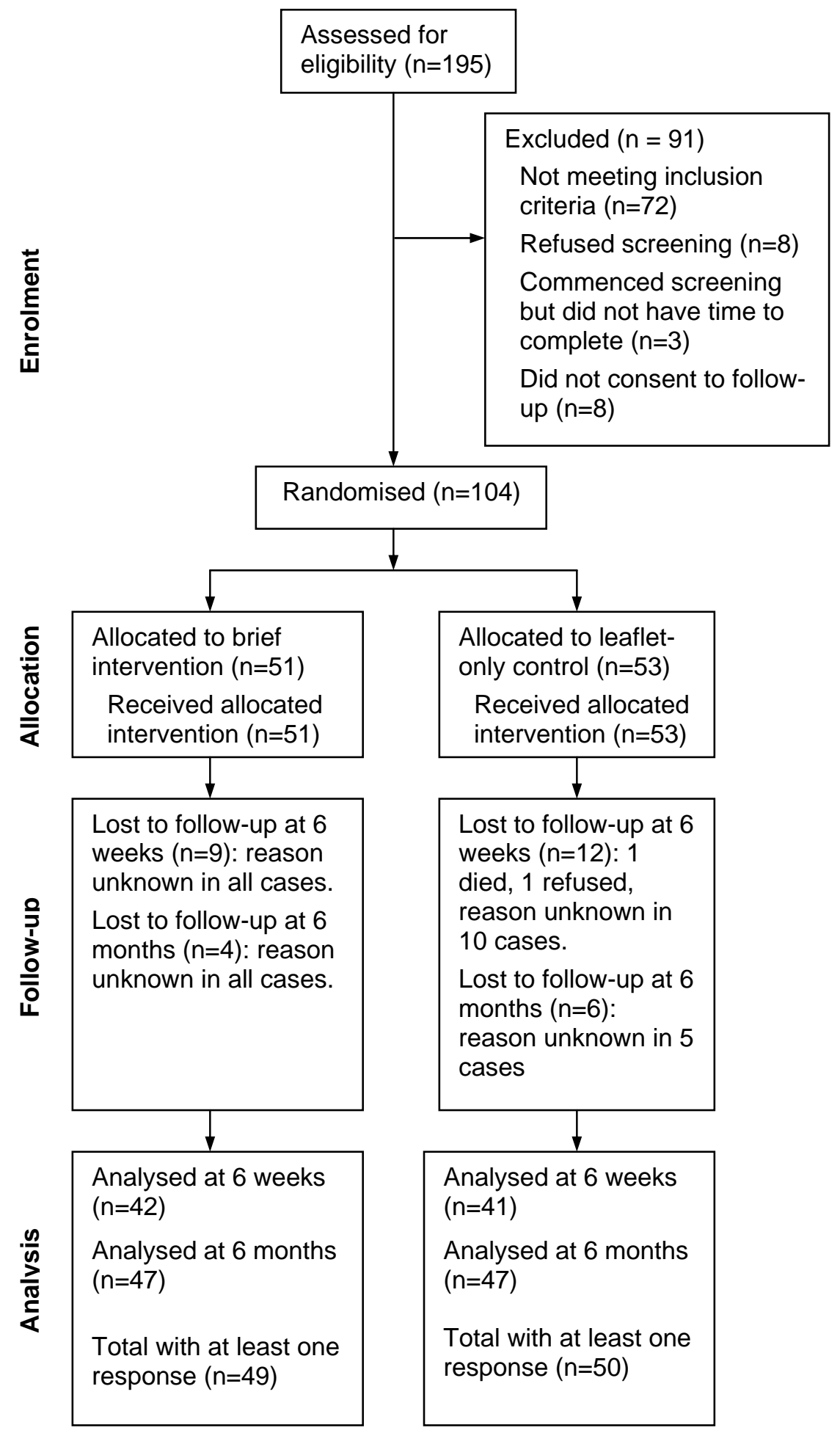

Figure 1. Trial profile 
In press 2004 - Addiction

Table 1. Age and AUDIT scores of the study groups

\begin{tabular}{lcccc}
\hline & \multicolumn{2}{c}{$\begin{array}{c}\text { Control } \\
(n=53)\end{array}$} & \multicolumn{2}{c}{$\begin{array}{c}\text { Intervention } \\
(n=51)\end{array}$} \\
& Mean & SD & Mean & SD \\
\hline Age & 20.4 & $(1.8)$ & 19.9 & $(1.4)$ \\
AUDIT score & 16.6 & $(6.0)$ & 16.6 & $(5.7)$ \\
\hline
\end{tabular}


In press 2004 - Addiction

Table 2. Summary of outcome variables at six-weeks and six-months post intervention

\begin{tabular}{|c|c|c|c|c|c|c|c|c|}
\hline \multirow{3}{*}{ Outcome } & \multicolumn{4}{|c|}{ Six weeks } & \multicolumn{4}{|c|}{ Six months } \\
\hline & \multicolumn{4}{|c|}{ Median (range) } & \multicolumn{4}{|c|}{ Median (range) } \\
\hline & \multicolumn{2}{|c|}{$\begin{array}{l}\text { Control } \\
(n=41)\end{array}$} & \multicolumn{2}{|c|}{$\begin{array}{l}\text { Intervention } \\
\qquad(\mathrm{n}=42)\end{array}$} & \multicolumn{2}{|c|}{$\begin{array}{l}\text { Control } \\
(n=47)\end{array}$} & \multicolumn{2}{|c|}{$\begin{array}{l}\text { Intervention } \\
\qquad(\mathrm{n}=47)\end{array}$} \\
\hline $\begin{array}{l}\text { 1. Frequency of drinking (number of drinking } \\
\text { days in last } 2 \text { weeks) }\end{array}$ & 4 & $(0-13)$ & 3 & $(0-9)$ & 4 & $(0-14)$ & 3 & $(0-8)$ \\
\hline $\begin{array}{l}\text { 2. Typical occasion quantity (number of } \\
\text { drinks* per typical drinking occasion in } \\
\text { last } 4 \text { weeks) }\end{array}$ & 9 & $(3-25)$ & 8 & $(2-20)$ & 8 & $(0-25)$ & 8 & $(0-20)$ \\
\hline $\begin{array}{l}\text { 3. Total consumption (number of drinks in } \\
\text { last } 2 \text { weeks) }\end{array}$ & 26 & $(0-152)$ & 24 & $(0-70)$ & 23 & $(0-125)$ & 26 & $(0-75)$ \\
\hline $\begin{array}{l}\text { 4. Frequency of very episodic heavy drinking } \\
\text { (number of episodes of }>80 \mathrm{~g} \text { for women } \\
\text { and } 120 \mathrm{~g} \text { for men in last } 2 \text { weeks) }\end{array}$ & 1 & $(0-7)$ & 1 & $(0-5)$ & 1 & $(0-12)$ & 1 & $(0-4)$ \\
\hline $\begin{array}{l}\text { 5. Personal, social, sexual, and legal } \\
\text { consequences of episodic heavy drinking } \\
\text { (number of problems - APS; range 0-14) }\end{array}$ & 3 & $(0-9)$ & 2 & $(0-7)$ & 3 & $(0-9)$ & 2 & $(0-8)$ \\
\hline $\begin{array}{l}\text { 6. Consequences related to tertiary student } \\
\text { role expectations (score on AREAS; } \\
\text { range } 0-35 \text { ) }\end{array}$ & 3 & $(0-18)$ & 1.5 & $(0-21)$ & 4 & $(0-17)$ & 2 & $(0-12)$ \\
\hline
\end{tabular}

* Standard drinks (10 g ethanol) 
Table 3. Six-week and six-month intervention effects, based on random effects models without imputation for missing values

\begin{tabular}{|c|c|c|c|}
\hline Outcome & $\begin{array}{c}\text { Ratio of } \\
\text { geometric means* } \\
\text { Intervention / Control }\end{array}$ & $(95 \% \mathrm{Cl})$ & $p$ \\
\hline \multicolumn{4}{|l|}{ 1. Frequency of drinking } \\
\hline 6 weeks & 0.80 & (0.63 to 1.02$)$ & 0.08 \\
\hline 6 months & 0.84 & (0.67 to 1.06$)$ & 0.15 \\
\hline \multicolumn{4}{|l|}{ 2. Typical occasion quantity } \\
\hline 6 weeks & 0.84 & (0.68 to 1.04$)$ & 0.11 \\
\hline 6 months & 1.02 & (0.81 to 1.27$)$ & 0.89 \\
\hline \multicolumn{4}{|l|}{ 3. Total consumption } \\
\hline 6 weeks & 0.74 & (0.56 to 0.96$)$ & 0.03 \\
\hline 6 months & 0.90 & (0.70 to 1.18$)$ & 0.46 \\
\hline \multicolumn{4}{|l|}{ 4. Frequency of very episodic heavy drinking } \\
\hline 6 weeks & 0.63 & $(0.42$ to 0.94$)$ & 0.02 \\
\hline 6 months & 0.85 & (0.59 to 1.22$)$ & 0.38 \\
\hline \multicolumn{4}{|l|}{$\begin{array}{l}\text { 5. Personal, social, sexual, and legal } \\
\text { consequences of episodic heavy drinking }\end{array}$} \\
\hline 6 weeks & 0.70 & (0.54 to 0.91$)$ & 0.01 \\
\hline 6 months & 0.76 & (0.60 to 0.97$)$ & 0.03 \\
\hline \multicolumn{4}{|l|}{$\begin{array}{l}\text { 6. Consequences related to tertiary student } \\
\text { role expectations }\end{array}$} \\
\hline 6 weeks & 0.74 & (0.53 to 1.03$)$ & 0.07 \\
\hline 6 months & 0.72 & (0.51 to 1.02$)$ & 0.06 \\
\hline Summary effect sizes ${ }^{\star \star}(95 \%$ Cls $)$ & 6 weeks & 6 months & \\
\hline Consumption measures (outcomes 1-4) & 0.40 & 0.15 & \\
\hline Alcohol-related problems (outcomes 5-6) & 0.45 & 0.44 & \\
\hline
\end{tabular}

* The exponent of the arithmetic mean of the log- transformed data.

** Positive values for effect sizes indicate better outcome for brief intervention compared with control. 